\title{
Efecto de la terapia de resincronización ventricular en los parámetros de disfunción endotelial y función sistólica izquierda en pacientes con insuficiencia cardíaca crónica y bloqueo completo de rama izquierda
}

\author{
Teresa Massardo ${ }^{1 a}$, Jaime Pereira ${ }^{2}$, Claudia G. Sáez. ${ }^{*}$, Ivonne Aramburú $^{1 b}$, Raimundo Morris ${ }^{1 b}$, Rubén Aguayo ${ }^{3}$, Solange Brugère $^{1 b}$, \\ Angela Pino ${ }^{1 b}$, Enrique Hiplan ${ }^{1 a}$, Gabriela Paillahueque ${ }^{1 a}$, Luis Alarcón ${ }^{1 a}$, Jane Spuler ${ }^{1 a}$, René Fernández ${ }^{1 a}$, Eduardo Swett1a \\ Eduardo Sanhueza $^{1 b}$, René Asenjo ${ }^{1 b}$, Macarena Palominos ${ }^{2 *}$, Nixa Olivares ${ }^{2 \#}$, Guillermo Valenzuela ${ }^{*}$, José Torres ${ }^{3}$, Javier Garate $^{4}$, \\ Christian Karmelic. 4
}

1. Hospital Clínico Universidad de Chile, 1a. Sección Medicina Nuclear, Dpto. Medicina, 1b. Departamento Cardiovascular. 2. Departamento de Hematología-Oncología, Escuela de Medicina, Pontificia Universidad Católica de Chile. 3. Departamento de cardiología, Hospital San Juan de Dios.

4. Departamento de cardiología, Hospital San Borja Arriarán. * Bioquímicos.

\# Bióloga, mención en bioprocesos.

Financiado por: Proyecto OIEA VISION CRP E1.30.

Introducción: La insuficiencia cardíaca crónica (ICC) es una condición compleja asociada a inflamación sistémica y a disfunción endotelial (DE) cuya patogénesis no es bien comprendida.

Objetivo: Evaluar una posible relación entre marcadores de DE periférica con la respuesta a terapia de resincronización ventricular (TRV).

Método: 20 pacientes con ICC, QRS $\geq 120 \mathrm{~ms}$ y fracción de eyección ventricular izquierda (FEVI) $\leq 35 \%$ se estudiaron pre y 6 meses post-TRV con: Minnesota Living with Heart Failure Questionnaire (MLHFQ); test de marcha (TM-6min); Ecocardiografía-2D y SPECT de perfusión gatillado en reposo; proteína C-reactiva ultra sensible (us-PCR); péptido natriurético cerebral (pro-BNP); células endoteliales circulantes (CEC); moléculas de adhesión soluble vascular (sVCAM) e intercelular (sICAM); interleukina-6 (IL-6) y Factor von Willebrand (FvW). Se clasificaron como respondedores o no a TRV según criterios preestablecidos.

Resultados: Promedios basales: pro-BNP 5.290 pg/ml; us-PCR 1,7ug/mL; MLHFQ 72; TM-6min 391 metros. Las CEC y sICAM estaban sobre límites normales. Post-TRV, el 50\% fue respondedor: 11/20 mejoraron $\geq 1$ clase NYHA y $\geq 10 \%$ del TM-6min; MLHFQ disminuyó $(\mathrm{p}<0.0001)$; FEVI mejoró $(\mathrm{p}=0.003)$; volumen final sistólico disminuyó ( $\mathrm{p}=0.008$ ) y también pro-BNP $(\mathrm{p}=0.03)$. En los respondedores, las CEC disminuyeron, persistiendo elevadas, sobre lo normal. Existieron correlaciones entre cambios de pro-BNP con TM-6min y entre us-PCR con MLHFQ y FvW ( $\mathrm{p} \leq 0.004$ en todas).

Conclusiones: En ICC existe evidencia de significativa DE, expresada por sICAM y CEC, biomarcador periférico sensible. Estas disminuyeron 6 meses postTRV, persistiendo sobre el límite normal. Otros parámetros funcionales e inflamatorios se correlacionaron en el grupo total, sin diferencias entre grupos respondedores y no respondedores.

Palabras clave: insuficiencia cardíaca; resincronización ventricular; disfunción endotelial.

\section{Correspondencia}

Dra. Teresa Massardo Vega.

Santos Dumont 999 1E, Independencia, Santiago de Chile.

tmassardo@hcuch.cl 


\section{Endothelial dysfunction, left ventricular function, and chronic heart failure with complete left bundle branch block: effect of resynchronization therapy}

Introduction: Chronic heart failure (CHF) is a complex condition associated with systemic inflammation and endothelial dysfunction (ED) whose pathogenesis is not well understood.

Objective: to evaluate a possible relationship between peripheral ED markers and response to cardiac resynchronization therapy (CRT).

Method: 20 patients with CHF, QRS $\geq 120 \mathrm{~ms}$ and left ventricular ejection fraction (LVEF) $\leq 35 \%$ were studied before and 6 months post-CRT. Minnesota Living with Heart Failure Questionnaire (MLHFQ); walking test (6min-WT); 2D-echocardiography and gated perfusion SPECT at rest; ultra-sensitive C-reactive protein (us-CRP); brain natriuretic peptide (proBNP); circulating endothelial cells (CEC); vascular soluble adhesion (sVCAM) and intercellular adhesion molecules (sICAM); interleukin-6 (IL-6) and von Willebrand Factor (vWF) were measured in all subjects. They were classified as responders or not to CRT, according to pre-established criteria.
Results: Basal means: pro-BNP 5,290 pg / ml; usCRP $1.7 \mathrm{ug} / \mathrm{mL}$; MLHFQ 72; 6min-WT 391 meters. The CEC and IL-6 were above normal limits. PostCRT, 50\% were responders: 11/20 improved $\geq 1$ NYHA class and $\geq 10 \%$ increase in 6min-WT; MLHFQ decreased ( $p<0.0001$ ); LVEF improved ( $p=0.003$ ); final systolic volume decreased $(\mathrm{p}=0.008)$ and also pro-BNP $(\mathrm{p}$ $=0.03$ ). In responders CEC decreased, persisting over normal limits. There were correlations between changes of pro-BNP with TM-6min and between us-PCR with MLHFQ and vWF ( $\mathrm{p} \leq 0.004$ in all).

Conclusions: In CHF there is evidence of significant ED, expressed by sICAM and CEC, a sensitive peripheral biomarker that decreased 6 months postCRT although persisting above normal limits. Other functional and inflammatory parameters were correlated in the total group, without differences between responders and non-responders.

Key words: Heart failure; ventricular resynchronization; endothelial dysfunction. 


\section{Introducción:}

La insuficiencia cardíaca crónica (ICC) se caracteriza por presentar diversas y complejas alteraciones entre las que se encuentran pérdida de cardiomiocitos, fibrosis y remodelación patológica, así como fenómenos de inflamación sistémica y disfunción endotelial (DE) cuya patogenia no es bien conocida. La DE es definida como la pérdida de las propiedades vasodilatadoras, antiadhesivas y antitrombóticas de las células endoteliales y se ha demostrado su existencia en pacientes portadores de diferentes patologías cardiovasculares ${ }^{1}$; se ha observado disminución en la biodisponibilidad de óxido nítrico en arterias periféricas y coronarias, por lo que ha sido propuesta como un predictor independiente de la evolución en pacientes con ICC $^{2}$. Recientemente, se ha descrito que la resincronización cardíaca se asocia a mejoría de la DE estudiada mediante vasodilatación mediada por flujo utilizando técnica ecográfica con compresión de arteria braquial, sin embargo, no se han evaluado marcadores circulantes de $\mathrm{DE}^{3}$. Entre estos, las células endoteliales circulantes (CEC) han mostrado ser un marcador sensible y específico en patologías inflamatorias asociadas a daño vascular tipo vasculitis ${ }^{4}$, usuarios de cocaína ${ }^{5,6}$ y depresión mayor ${ }^{7,8}$.

Por otra parte, el péptido natriurético cerebral (pro-BNP) ha sido utilizado para evaluar la ICC en distintas condiciones y es interesante conocer su comportamiento con relación a la terapia de resincronización ventricular $(\mathrm{TRV})^{9,}, 10$ y asociación con la DE. Otros marcadores inflamatorios que se han encontrado alterados en la ICC son la interleukina-6 (IL-6) y el factor de necrosis tumoral-alfa (TNF $\alpha)$. El pro-BNP y la IL-6 se han considerado útiles como biomarcadores en ICC $^{11}$; el TNF $\alpha$ estaría involucrado en su patogenia ${ }^{12,13}$.

No todos los pacientes sometidos a TRV responden en la misma forma a esta terapia a pesar de una correcta indicación y ejecución del procedimiento de implantación de electrodos; en este sentido, sería de gran utilidad disponer de predictores seguros de respuesta a TRV pues al menos $30 \%$ de los casos, en la actualidad, no presentan mejoría. Los respondedores a TRV logran mejor sobrevida, calidad de vida y capacidad de ejercicio, debido a una mayor sincronía en la contracción mecánica ventricular. La mejoría de función sistólica izquierda medida con fracción de eyección (FEVI) y el volumen ventricular, especialmente de final de sístole (VFS), son los parámetros más considerados para definir la respuesta, aunque se usan también la capacidad funcional y la calidad de vida $^{14-20}$.
En el contexto de un proyecto para evaluar la sincronía del ventrículo izquierdo con método isotópico y predecir éxito del procedimiento de resincronización en ICC, consideramos interesante estudiar un subgrupo de pacientes locales con marcadores de DE e inflamación y evaluar sus potenciales cambios con la TRV.

Objetivo principal: evaluar la relación entre marcadores de DE periféricos y parámetros funcionales cardíacos en pacientes con ICC y bloqueo completo de rama izquierda (BCRI) sometidos a TRV con control a 6 meses.

\section{Material y Métodos:}

Se estudiaron 20 pacientes chilenos, como parte de un proyecto multicéntrico internacional de investigación coordinado del Organismo Internacional de Energía Atómica (OIEA), con edad promedio 64 años (rango: 43-77 años), 8 mujeres y 12 hombres: todos con ICC en clase funcional entre II y IV NYHA; 9/20 con enfermedad coronaria demostrada por coronariografía de contraste, 4 con antecedentes de infarto al miocardio y 6 de revascularización.

Los criterios de inclusión fueron los siguientes: requerimiento de resincronizador, pacientes estables, adultos, refractarios a terapia médica de ICC optimizada acorde a las guías vigentes por al menos 3 meses, FEVI $\leq 35 \%$ y QRS >120 ms con morfología de BCRI y ritmo sinusal. Los criterios de exclusión eran, entre otros: bloqueo completo de rama derecha, fibrilación o flutter auricular. Se aceptaba la planificación de desfibrilador automático.

Basalmente y 6 meses post-TRV se efectuaron ecocardiografía-2D, encuesta de calidad de vida con cuestionario Minnesota Living with Heart Failure Questionnaire (MLHFQ) y prueba de marcha de 6 minutos (TM-6min), con determinación de oximetría, parámetros de presión arterial y frecuencia cardíaca.

Paralelamente, el mismo día se adquirió un SPECT gatillado con sestamibi Tc99m en reposo evaluando función sistólica y sincronismo de contracción ventricular izquierda.

Los pacientes se clasificaron como respondedores a TRV, de acuerdo con el consenso previo del proyecto si cumplían al menos 3 de los siguientes 4 parámetros: aumento FEVI $\geq 5 \%$, disminución de volumen final sistólico $\geq 15 \%$, mejoría de CF NYHA $\geq 1$ grado y mejoría $\geq 5$ puntos en MLHFQ.

Todos los pacientes firmaron consentimiento informado del comité de ética científico de la Institución, de acuerdo con protocolo de Helsinki, con aprobación del proyecto 
de parte de los hospitales referentes. Los pacientes provenían de los Hospitales Clínico de la Universidad de Chile, San Juan de Dios y San Borja Arriarán. Se efectuó seguimiento clínico a todos los casos a los 12 meses con entrevista telefónica, consulta con su tratante y de fichas clínicas disponibles.

Las imágenes ecocardiográficas 2D en reposo se efectuaron con equipo Philips IE 33 inmediatamente antes o después de los SPECT y el mismo día que las muestras sanguíneas. Se evaluaron FEVI y volúmenes de ventrículo izquierdo con método de Simpson y diámetro diastólico basal de ventrículo derecho, Excursión anular tricuspídea sistólica (TAPSE) y onda s tisular de la válvula tricúspide, para función de ventrículo derecho.

Los estudios isotópicos se efectuaron en reposo pre y 6 meses post -TRV. Se inyectaron $740 \mathrm{MBq}$ de sestamibi (marcación del radiofármaco sobre 90\%). A la hora, se efectuó adquisición con cámara Siemens e.cam doble cabezal, en órbita $180^{\circ}$, gatillado con 8 frames, zoom:1, 2, matrix $64 \times 64$. El procesamiento fue habitual, con método iterativo. El análisis de perfusión y función miocárdica se efectuó con software Cedars Sinai. El análisis de sincro- nismo de la contracción ventricular se realizó mediante el software Synctool ${ }^{\circledR}$ de Emory Toolbox, obteniendo parámetros de fase basado en análisis de Fourier correspondientes a desviación estándar (SD) y ancho de banda (BW) del histograma de contracción en el ciclo cardiaco sumado. El valor que se ha publicado para SD promedio normal es de $15.3^{\circ}$ y se considera como valor de corte alto para disincronía significativa $S D \geq 43^{\circ}$ y $B W \geq 135^{\circ}$. Nuestros valores mostraban distintos grados de disincronía. Se eliminó un caso para este análisis pues presentó arritmia transitoria durante la adquisición gatillada inicial $^{21-25}$.

-Mediciones de laboratorio: Se midieron en sangre periférica marcadores inespecíficos de inflamación como proteína $\mathrm{C}$ reactiva ultrasensible (us-PCR) e IL-6, proBNP, así como marcadores de DE específicos mediante recuento de CEC; asimismo, se determinó el nivel de marcadores solubles de daño/activación endotelial como moléculas solubles de adhesión celular vascular (sVCAM), e intercelular (sICAM) y el factor von Willebrand (FvW). Para las determinaciones, la sangre se obtuvo en ayunas

Tabla 1: Principales parámetros basales, pre-TRV.

\begin{tabular}{|c|c|c|c|}
\hline Parámetro & n & Promedio & Rango \\
\hline Edad (años) & 64.2 & 43-77 & \\
\hline Sexo masculino & $12 / 20$ & & \\
\hline IMC (kg/m2) & 28 & $20-38$ & \\
\hline QRS (ms) & 166 & $138-200$ & \\
\hline Clase NYHA: II & $9 / 20$ & & \\
\hline III & $8 / 20$ & & \\
\hline IV & $3 / 20$ & & \\
\hline FEVI SPECT manual Cedars (\%) & & 21 & $8-38$ \\
\hline FEVI ECO Simpson (\%) & & 22 & $9-34$ \\
\hline VFS Simpson (ml) & & 162 & $81-379$ \\
\hline Diámetro diastólico VI (mm) & & 64 & $52-74$ \\
\hline Diámetro diastólico VD basal (mm) & & 42.1 & $32-53$ \\
\hline Summed Rest Score SRS Cedars & & 15 & 3-37 \\
\hline Excentricidad izquierda SPECT Cedars & & 0.77 & $0.68-0.84$ \\
\hline Masa gatillada izquierda Emory ToolBox (gr) & & 232 & $181-321$ \\
\hline TM-6min (\% esperado para edad) & & 74.4 & $12-110$ \\
\hline Histograma de fase SD $\left({ }^{\circ}\right)$ & & 55 & $11-302$ \\
\hline Histograma de fase BW $\left(^{\circ}\right)$ & & 134 & $39-360$ \\
\hline MLHFQ (puntos) & & 71 & $36-93$ \\
\hline pro-BNP (pg/mL) & & 5290 & $481-18200$ \\
\hline
\end{tabular}

TM-6min: test de marcha de 6 min; MLHFQ: Minnesota Living with Heart Failure Questionnaire; VFS: Volumen de fin de sístole izquierdo. 
entre 9 y 10 AM mediante punción de la vena antecubital con aguja 19-G sin estasis venoso. Para las CEC se usaron tubos de $5 \mathrm{ml}$ con anticoagulante EDTA; para los marcadores solubles, sangre anticoagulada con citrato de sodio al 3.2\%. La enumeración de CEC se realizó mediante captura de éstas con inmunoesferas magnéticas cubiertas con anticuerpo anti-CD146 y microscopía de fluorescencia según protocolo del laboratorio previamente descrito ${ }^{5}$. Los niveles de sVCAM, sICAM y FvW se determinaron mediante ELISA (Bender Med Systems). Para el marcador humoral de insuficiencia cardíaca $\mathrm{N}$ terminal pro-BNP se requirieron $5 \mathrm{ml}$ adicionales de plasma y se empleó la prueba inmunométrica Vitros MicroWELL ${ }^{\mathrm{TM}}$.

-Análisis estadístico: se calcularon las diferencias de los promedios con prueba " $t$ " de Student y correlaciones de los cambios de los datos pareados según normalidad con Pearson o Spearman $(\mathrm{p}<0.05)$.

\section{Resultados:}

En condiciones basales, los valores promedio de proBNP, MLHFQ score y TM-6min se encontraban alterados respecto a lo normal (Tabla 1). La evaluación de los parámetros de DE mostró que las CEC estaban muy aumentadas; en cuanto a los marcadores solubles, se observó un aumento significativo de sICAM (Tabla 2).

La resincronización se efectuó en todos sin conocimiento de los datos de sincronismo ventricular, en 2 de los casos con técnica epicárdica; se implantaron 6 desfibriladores automáticos, en forma concomitante.

Post-TRV, respecto al valor basal, el grupo completo mostró disminución del pro-BNP $(\mathrm{p}=0.03)$ y tendencia

\begin{tabular}{l|l|c|c|c|}
$\begin{array}{l}\text { Tabla } 2 \\
\text { Marcadores de inflamación y disfunción endotelial basales en grupo } \\
\text { completo. }\end{array}$ \\
\hline Marcador & Promedio & Rango & Control normal & Valor p \\
\hline us-PCR (ug/mL) & 1.70 & $0.22-3.97$ & $0.3 \pm 0.62$ & n.s. \\
\hline IL-6 (pg/ml) & 10 & $4.3-38.8$ & $1.2 \pm 3.6$ & n.s. \\
\hline sICAM (ng/ml) & 203 & $100-385$ & $156 \pm 50$ & 0.03 \\
\hline sVCAM (ng/ml) & 579.8 & $316-986$ & $486 \pm 61$ & n.s. \\
\hline FvW (\%) & 158.1 & $90-341$ & $98 \pm 25$ & n.s. \\
\hline CEC (células/ml) & 68 & $32-102$ & $9.3 \pm 3.6$ & 0.0001 \\
\hline
\end{tabular}

us-PCR: proteína C reactiva ultrasensible; IL-6: interleukina 6; slCAM: moléculas de adhesión soluble intercelular; sVCM: moléculas de adhesión soluble intra vascular; FvW: factor de von Willebrand; CEC: células endoteliales circulantes. a disminución de us-PCR ( $\mathrm{p}=\mathrm{ns})$. El MLHFQ disminuyó de 71 a 45 puntos en promedio ( $\mathrm{p}<0.0001$ ). El TM-6min no varió significativamente. La excentricidad del ventrículo izquierdo que representa remodelación patológica y la masa gatillada disminuyeron $(\mathrm{p}=0.02)$, persistiendo la primera sobre límites normales ${ }^{26}$.

Por otra parte, en el grupo completo, se observó correlación de los cambios entre basal y control de 6 meses de pro-BNP con TM-6min ( $\mathrm{r}=0.617$; $\mathrm{p}=0.004)$ y también de correlación de los cambios de us-PCR con puntaje de MLHFQ ( $\mathrm{r}=0.617 ; \mathrm{p}=0.004)$ y con FvW ( $\mathrm{r}=0.663$; $\mathrm{p}=0.002$ ).

El 50\% de los pacientes fue respondedor a TRV según los criterios preestablecidos; 11/20 pacientes aumentaron al menos 1 clase funcional NYHA y $\geq 10 \%$ de su TM- 6 min. No hubo diferencias de edad, de proporción de enfermedad coronaria, diabetes mellitus ni hipertensión arterial entre subgrupos $(\mathrm{p}=\mathrm{ns})$.

En los respondedores a TRV, el puntaje MLHFQ score disminuyó ( $\mathrm{p}<0.0001)$, la FEVI mejoró ( $\mathrm{p}=0.003$ ) y también el VFS $(\mathrm{p}=0.008)$. No hubo cambio en la capacidad de marcha.

En casi todos los casos hubo alteración de perfusión septal explicable por presencia de BCRI. En los no respondedores, el índice del defecto de perfusión en reposo Summed Rest Score (SRS) aumentó ( $\mathrm{p}=0.008$ ); la disincronía no cambió en forma significativa. En ambos subgrupos, el pro-BNP disminuyó significativamente (excluyendo 1 caso en cada uno en que hubo marcada elevación). A nivel de ventrículo izquierdo, no hubo diferencias en la excentricidad ni masa gatillada, ni tampoco en diámetro diastólico; no mejoraron el grado de insuficiencia mitral ni el diámetro de ventrículo derecho (medido a nivel basal) ni la proporción de disfunción ventricular derecha $(\mathrm{p}=\mathrm{ns})$. Las diferencias significativas entre respondedores y no respondedores se muestran en la Tabla 3 .

A los 12 meses de seguimiento clínico, no hubo muertes ni eventos cardiovasculares, sólo un accidente vascular encefálico, que alteró la prueba de marcha.

\section{Discusión:}

En el grupo respondedor a TRV, el pro-BNP, biomarcador sistémico de ICC, la FEVI y el MLHFQ disminuyeron significativamente; sin embargo, no mejoraron la capacidad de marcha, ni la dilatación ventricular derecha; no hubo eventos cardíacos mayores al año de seguimiento. Con los criterios usados, el porcentaje respondedor fue algo menor en tamaño que lo publicado en la literatura y no se observó mejoría en la sincronía de contracción 


\begin{tabular}{|l|c|c|c|c|c|c|}
\hline $\begin{array}{l}\text { Tabla } 3 \\
\text { Parámetros promedio con significancia estadística en respondedores y no } \\
\text { respondedores a Terapia de Resincronización Ventricular (TRV), respecto a su basal. }\end{array}$ \\
\hline & \multicolumn{3}{|c|}{ Respondedor } & \multicolumn{3}{r|}{ No Respondedor } \\
\hline Parámetro & Pre TRV & Post TRV & p & Pre TRV & Post TRV & p \\
\hline CEC (n /ml) & 64 & 47 & 0.019 & 71 & 56 & ns \\
\hline MLHFQ (puntos) & 68 & 39 & 0.0002 & 55 & 72 & 0.0156 \\
\hline FEVI Simpson (\%) & 22.3 & 34.8 & 0.002 & 22.7 & 25.2 & ns \\
\hline VFS Simpson (ml) & 163 & 125 & $<0.0001$ & 161 & 157 & ns \\
\hline SRS & 13 & 14 & ns & 15 & 19 & 0.008 \\
\hline pro-BNP & 5228 & $3912^{*}$ & 0.044 & 5352 & 5177 & $0.008 \#$ \\
\hline
\end{tabular}

CEC: células endoteliales circulantes; MHLFQ: Minnesota Living with Heart Failure Questionnaire FEVI: fracción de eyección de VI; VFS: volumen sistólico final de VI; SRS: summed rest score; pro-BNP: péptido natrurético cerebral.

*,\# valor post-TRV muy aumentado excluido de análisis Student (color, Figura 3).

izquierda, tal vez debido al pequeño tamaño de la muestra local. En los resultados preliminares de la muestra completa multicéntrica ${ }^{27}$ que contó con 169 pacientes, 17 fallecieron antes del control a los 6 meses y del resto, el $77 \%$ logró uno o más de los 4 outcomes primarios aislados. En nuestro trabajo local, fuimos más exigentes requiriendo al menos 3 criterios de respuesta, como fue acordado inicialmente. La definición de respondedor o no a TRV es relativamente controvertida y probablemente requiere mayor seguimiento que 6 meses.

Como era de esperar, el pro-BNP basal estuvo muy elevado en casi todos nuestros pacientes; los valores normales varían según sexo y aumentan con la edad; a los 6 meses post-TRV, tanto respondedores, como no-respondedores, persistieron con pro-BNP elevado. Sin embargo, en ambos grupos se observó una disminución significativa, salvo en un caso en cada grupo en quienes el proBNP empeoró (Figura 3). El valor máximo aceptable de pro-BNP en nuestro grupo según edad es bajo $200 \mathrm{pg} / \mathrm{ml}$, pues no tuvimos mujeres mayores de 75 años.

Se ha descrito que a los 3 meses post terapia médica optimizada, el pro-BNP y la IL-6 son factores independientes para predecir mortalidad, a pesar de mejoría de FEVI y síntomas ${ }^{11}$. El us-PCR, un marcador muy inespecífico de inflamación, presentó elevación leve en sólo algunos casos y no tuvo cambios significativos.

Con respecto a la evaluación de DE, encontramos valores muy elevados de las CEC, que se han considerado como un parámetro muy sensible y reproducible.
El aumento en los niveles de sICAM-1, comparados a los controles del laboratorio ${ }^{28}$, es también evidencia de daño endotelial. Es particularmente interesante que el número de CEC disminuyó significativamente después de la TRV, lo que apoya la hipótesis que en la ICC, la DE juega un papel importante en el proceso. Sin embargo, no encontramos diferencias significativas en los valores de CEC basales entre pacientes respondedores y no respondedores, lo que limita su valor como predictor de respuesta a TRV.

Es interesante mencionar que un grupo de ICC tratados con trasplante cardíaco ${ }^{29}$ mostró que otro marcador soluble de DE como la sVCAM-1 también se mantuvo elevada y disminuyó a los 2 años post-procedimiento. Por otra parte, también se demostró en esos casos que la elevación persistente de la P-selectina soluble (marcador de activación plaquetaria) y la falta de normalización de la sVCAM-1 estaba asociada con niveles persistentemente altos de $\mathrm{TNF} \alpha$, sin relación con episodios de infecciones intercurrente, ni de rechazo al injerto, apoyando la idea que los procesos inmunológicos e inflamatorios son importantes en la ICC. Por esta razón, debieran considerarse también otras citokinas pro-inflamatorias en la respuesta a TRV ${ }^{12,30}$. Se ha descrito, además, que los monocitos circulantes derivados de células progenitoras de endotelio están también aumentados en ICC, con diferencias étnicas; las progenitoras de endotelio también están alteradas en esta condición ${ }^{31,32}$.

Observamos correlaciones positivas entre cambios de pa- 


\section{Figura 1}

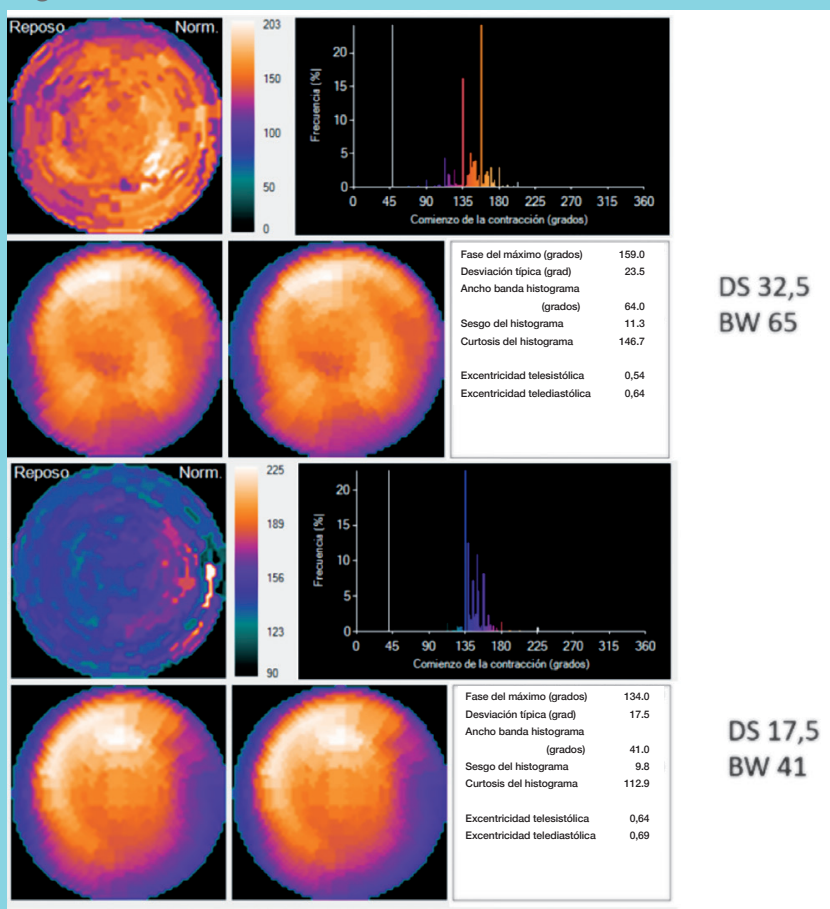

Sincronía de contracción mecánica del ventrículo izquierdo expresada en grados; en las imágenes superiores, paciente en condiciones basales y en las inferiores, 6 meses post TRV, se observa histograma de pixeles en contracción más angosto, de un paciente respondedor.

Figura 2

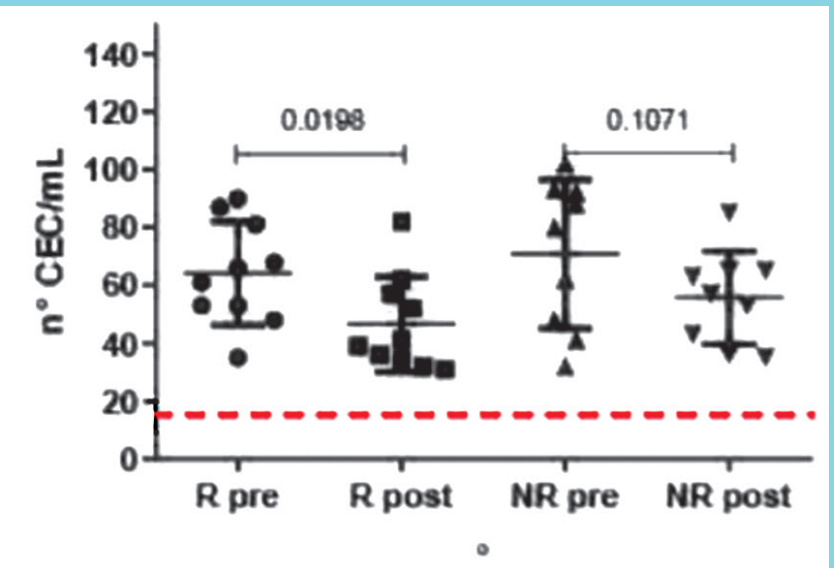

Respuesta de las CEC a la TRV en pacientes respondedores (R) con disminución post terapia y no respondedores (NR), sin cambios. La línea roja punteada representa el promedio +2 desviaciones estándar de nuestros controles normales.

rámetros de función ventricular y capacidad funcional, pero sin diferencias entre subgrupos respondedores y no respondedores, probablemente debido al pequeño tama- ño muestral. La existencia de defecto de perfusión en reposo de predominio septal en casos sin infarto es explicable por la presencia de BCRI. El aumento del defecto de perfusión septal observado en los no respondedores no es fácil de explicar pues en nuestros casos la sincronía de contracción ventricular no empeoró.

La definición de respondedores en este trabajo fue muy estricta, sin embargo, otros autores recientemente han usado criterios similares para evaluar la DE como respuesta sistémica a $\mathrm{TRV}^{3}$ clasificando respondedores a terapia a los 12 meses con parámetros de FEVI, VFS, clase funcional NYHA y TM-6min utilizando vasodilatación mediada por flujo a nivel braquial. Esos autores plantean que la DE no es un predictor independiente de TRV pero sí sería capaz de pesquisar sus efectos sistémicos como un marcador asequible para evaluar respuesta, especialmente en sujetos con dificultad o imposibilidad de efectuar prueba de marcha adecuada.

En relación a la fibrosis miocárdica que se puede evidenciar con resonancia cardiaca magnética, se ha reportado que a mayor fibrosis se observa mayor proporción de no respondedores a TRV 33 . En este sentido, la galectina-3 se ha descrito como nuevo biomarcador plasmático predictor de respuesta a TRV ya que evidencia la fibrosis; este fenómeno estaría más relacionado con inflamación y remodelación reversa. El implantar el electrodo ventricular izquierdo en un segmento miocárdico fibrótico o infartado impide mejorar la sincronía contráctil; la visualización del último segmento viable que se contrae, mediante SPECT, sería un elemento de apoyo que permitiría optimizar la implantación, pronóstico y evaluación del efecto de la TRV 23,34 .

Un trabajo reciente plantea que los niveles basales de DE mediante método de flujo de arteria braquial podrían utilizarse para predecir respuesta 6-12 meses postTRV, basado sólo en los mecanismos dependientes del endotelio. Esos autores contaban con 14 respondedores y 5 no respondedores ${ }^{35}$. Además, los marcadores de DE pudieran ser utilizados como marcadores de la ICC con FEVI conservada, una nueva entidad de complejo manejo, lo cual fue planteado también con método de arteria braquial ${ }^{36}$. Otro método periférico que se ha descrito es la tonometría digital que parece tener buena correlación con la medición clásica de DE mediada por flujo en $\mathrm{EC}^{37,38}$.

Los resultados obtenidos en este trabajo muestran que los pacientes con ICC presentan aumento en marcadores de DE; el mecanismo que media este daño endotelial se desconoce. Sin embargo, en otras patologías asociadas a 


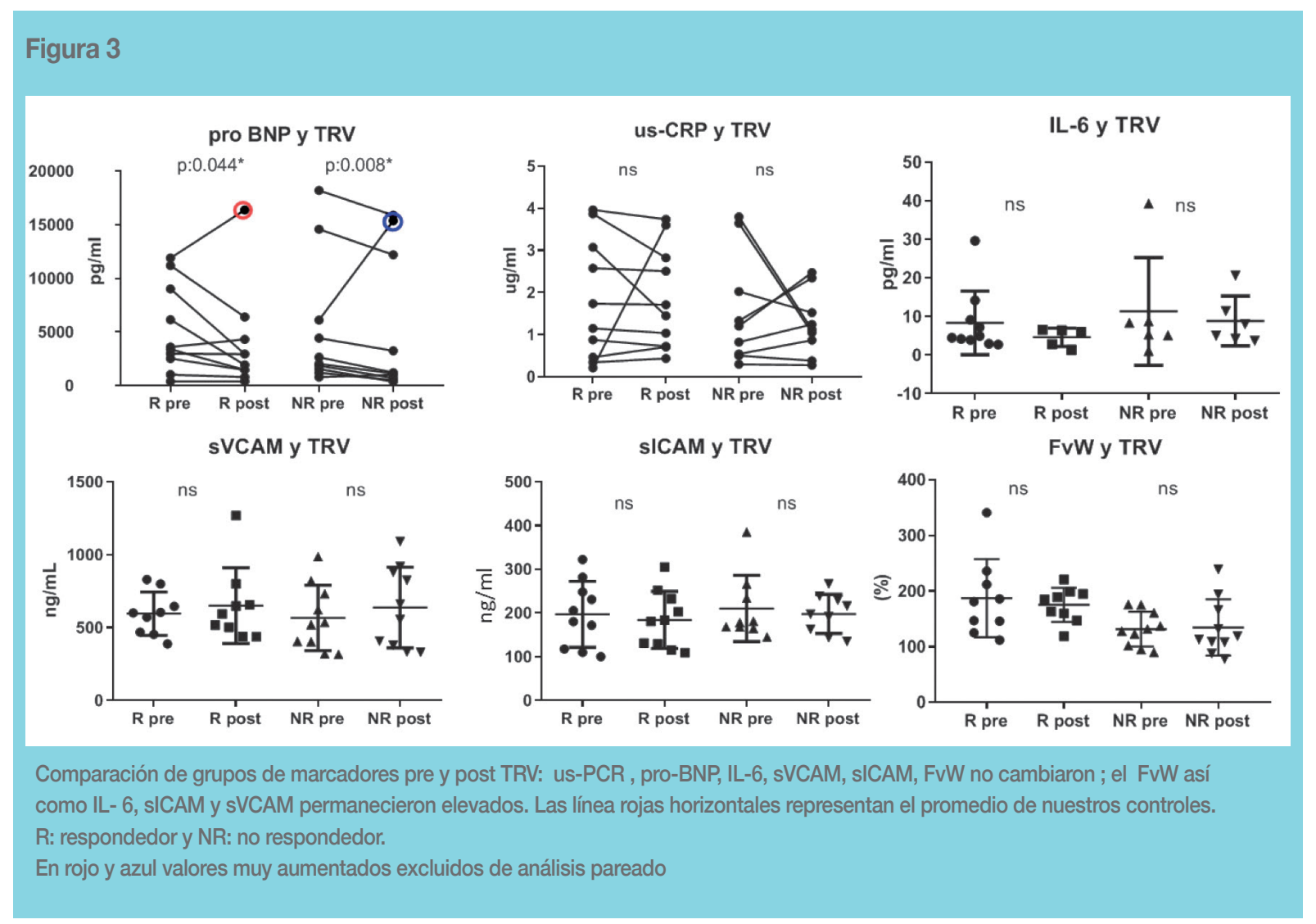

daño vascular como el síndrome metabólico o uso crónico de cocaína ${ }^{6,28}$ hemos demostrado que la activación de la via RhoA/Rho kinasa juega un papel importante en su génesis. Existe evidencia de que la actividad de Rho-kinasa está muy aumentada en pacientes con ICC estable, controlada médicamente, y que además, se encuentra asociada a remodelamiento del ventrículo izquierdo y disfunción sistólica ${ }^{39}$ : se la considera como un nuevo marcador de insuficiencia cardíaca. Por lo tanto, queda abierta para una eventual futura investigación el estudio de la activación de la vía RhoA/Rho-kinasa y sus potenciales cambios con TRV.

Una fortaleza de los datos presentados es que, a pesar del pequeño número de sujetos reclutados, se logró demostrar alteración y cambio en los marcadores de DE, especialmente las CEC, en los grupos de respondedores a TRV. La existencia de DE importante en este tipo de pacientes con ICC refractaria podría abrir un nuevo blanco terapéutico dirigido a la protección del endotelio, como parte de su manejo integral. 


\section{Referencias}

1 HIGASHI Y, NOMA K, YOSHIZUMI M, KIHARA Y. Endothelial function and oxidative stress in cardiovascular diseases. Circ J. 2009; 73: 411-8.

2 DE BERRAZUETA JR, GUERRA-RUIZ A, GARCIA-UNZUETA MT, TOCA GM, LASO RS, DE ADANA MS, et al. Endothelial dysfunction, measured by reactive hyperaemia using strain-gauge plethysmography, is an independent predictor of adverse outcome in heart failure. European Journal of Heart Failure. 2010; 12: 477-83. DOI 10.1093/eurjhf/ hfq036.

3 SANTINI L, CAPRIA A, DI MOLFETTA A, MAHFOUZ K, PANATTONI G, MINNI V, et al. Endothelial dysfunction is a marker of systemic response to the cardiac resynchronization therapy in heart failure. J Card Fail. 2013; 19: 419-25. 10.1016/j.cardfail.2013.05.001.

4 HAUBITZ M, DHAYGUDE A, WOYWODT A. Mechanis$\mathrm{ms}$ and markers of vascular damage in ANCA-associated vasculitis. Autoimmunity. 2009; 42: 605-14.

5 MASSARDO T, QUINTANA JC, JAIMOVICH R, SAEZ CG, CABRERAS MJ, PEREIRA-FLORES K, et al. Changes in regional cerebral blood flow are associated with endothelial dysfunction markers in cocaine-dependent patients under recent abstinence. J Addict Med. 2015; 9: 139-46. 10.1097/ADM.0000000000000109.

6 SAEZ CG, OLIVARES P, PALLAVICINI J, PANES O, MORENO N, MASSARDO T, et al. Increased number of circulating endothelial cells and plasma markers of endothelial damage in chronic cocaine users. Thromb Res. 2011; 128: e18-23. 10.1016/j.thromres.2011.04.019.

7 JAIMOVICH R MT, SAEZ C, QUINTANA J, RISCO L, GALLEGUILLOS T, ARAYA V, LIBERMAN C, CASTRO G, PEREIRA J. SPECT evaluation of regional cerebral blood flow in major depressive disorder: Effect of therapy and relationship with endothelial dysfunction markers. . J Nucl Med. 2011; 521.

8 LOPEZ-VILCHEZ I, DIAZ-RICART M, NAVARRO V, TORRAMADE S, ZAMORANO-LEON J, LOPEZ-FARRE A, et al. Endothelial damage in major depression patients is modulated by SSRI treatment, as demonstrated by circulating biomarkers and an in vitro cell model. Transl Psychiatry. 2016; 6: e886. 10.1038/tp.2016.156.

9 TROUGHTON R MFG, JANUZZI JL JR. Natriuretic peptide-guided heart failure management. Eur Heart J. 2014 35: 9.

10 HOOGSLAG GE, HOKE U, THIJSSEN J, AUGER D, MARSAN NA, WOLTERBEEK R, et al. Clinical, echocardiographic, and neurohormonal response to cardiac resynchronization therapy: are they interchangeable? Pacing Clin Electrophysiol. 2013; 36: 1391-401. 10.1111/pace.12214.

11 MAEDA K, TSUTAMOTO T, WADA A, MABUCHI N, HAYASHI M, TSUTSUI T, et al. High levels of plasma brain natriuretic peptide and interleukin- 6 after optimized treatment for heart failure are independent risk factors for morbidity and mortality in patients with congestive heart failure. J Am Coll Cardiol. 2000; 36: 1587-93.

12 FELDMAN AM, COMBES A, WAGNER D, KADAKOMI T, KUBOTA T, LI YY, et al. The role of tumor necrosis factor in the pathophysiology of heart failure. J Am Coll Cardiol. 2000; 35: 537-44.

13 MCTIERNAN CF, FELDMAN AM. The role of tumor necrosis factor alpha in the pathophysiology of congestive heart failure. Curr Cardiol Rep. 2000; 2: 189-97.

14 AURICCHIO A, DING J, SPINELLI JC, KRAMER AP, SALO RW, HOERSCH W, et al. Cardiac resynchronization therapy restores optimal atrioventricular mechanical timing in heart failure patients with ventricular conduction delay. $\mathrm{J}$ Am Coll Cardiol. 2002; 39: 1163-9.

15 AURICCHIO A, STELLBRINK C, SACK S, BLOCK M, VOGT J, BAKKER P, et al. Long-term clinical effect of hemodynamically optimized cardiac resynchronization therapy in patients with heart failure and ventricular conduction delay. J Am Coll Cardiol. 2002; 39: 2026-33.

16 CAZEAU S, LECLERCQ C, LAVERGNE T, WALKER S, 
VARMA C, LINDE C, et al. Effects of multisite biventricular pacing in patients with heart failure and intraventricular conduction delay. N Engl J Med. 2001; 344: 873-80.

17 ABRAHAM WT, FISHER WG, SMITH AL, DELURGIO DB, LEON AR, LOH E, et al. Cardiac resynchronization in chronic heart failure. N Engl J Med. 2002; 346: 1845-53.

18 YOUNG JB, ABRAHAM WT, SMITH AL, LEON AR, LIEBERMAN R, WILKOFF B, et al. Combined cardiac resynchronization and implantable cardioversion defibrillation in advanced chronic heart failure: the MIRACLE ICD Trial. JAMA. 2003; 289: 2685-94.

19 BRISTOW MR, SAXON LA, BOEHMER J, KRUEGER S, KASS DA, DE MARCO T, et al. Cardiac-resynchronization therapy with or without an implantable defibrillator in advanced chronic heart failure. N Eng1 J Med. 2004; 350: 2140-50.

20 BREATHETT K, ALLEN LA, UDELSON J, DAVIS G, BRISTOW M. Changes in Left Ventricular Ejection Fraction Predict Survival and Hospitalization in Heart Failure With Reduced Ejection Fraction. Circ Heart Fail. 2016; 9. 10.1161/CIRCHEARTFAILURE.115.002962.

21 CHEN J, GARCIA EV, LERAKIS S, HENNEMAN MM, BAX JJ, TRIMBLE MA, et al. Left ventricular mechanical dyssynchrony as assessed by phase analysis of ECG-gated SPECT myocardial perfusion imaging. Echocardiography. 2008; 25: 1186-94. 10.1111/j.1540-8175.2008.00782.x.

22 CHEN J, GARCIA EV, FOLKS RD, COOKE CD, FABER TL, TAUXE EL, et al. Onset of left ventricular mechanical contraction as determined by phase analysis of ECG-gated myocardial perfusion SPECT imaging: development of a diagnostic tool for assessment of cardiac mechanical dyssynchrony. J Nucl Cardiol. 2005; 12: 687-95. 10.1016/j.nuclcard.2005.06.088.

23 CHEN J, GARCIA EV, HENNEMAN MM, BAX JJ, BOOGERS MJ, TRIMBLE MA, et al. Measuring left ventricular mechanical dyssynchrony from ECG-gated SPECT myocardial perfusion imaging. Minerva Cardioangiol. 2008; 56: 227-35

24 HENNEMAN MM, CHEN J, DIBBETS-SCHNEIDER P, STOKKEL MP, BLEEKER GB, YPENBURG C, et al. Can LV dyssynchrony as assessed with phase analysis on gated myocardial perfusion SPECT predict response to CRT? J Nucl Med. 2007; 48: 1104-11. 10.2967/jnumed.107.039925.
25 HENNEMAN MM, CHEN J, YPENBURG C, DIBBETS P, BLEEKER GB, BOERSMA E, et al. Phase analysis of gated myocardial perfusion single-photon emission computed tomography compared with tissue Doppler imaging for the assessment of left ventricular dyssynchrony. J Am Coll Cardiol. 2007; 49: 1708-14. 10.1016/j.jacc.2007.01.063.

26 MASSARDO T BI, CASTRO G, MUÑOZ MP, SANTIS N, PADILLA P, PRAT H, ARAYA VA. Valor del índice de excentricidad del ventrículo izquierdo con tomografía SPECT de perfusión miocárdica en diversas condiciones. Rev Chil Cardiol. 2011; 31: 8 .

27 A. PEIX TM, G. KARTHIKEYAN, M. KALAIVANI, C. PATEL,M. PABON, A. JIMÉNEZ-HEFFERNAN, E. ALEXANDERSON, S. BUTT, A. KUMAR, V. MARIN, C. TINOCO MESQUITA,1 E. GARCIA, O. MOROZOVA, D. PAEZ. . Left Ventricular Dyssynchrony Improvement as Assessed by SPECT Myocardial Perfusion Imaging Predicts Clinical Outcomes in Heart Failure Patients Undergoing Cardiac Resynchronization Therapy (CRT): a Multi-National Prospective Trial. . J Nucl Cardiol. 2018; 251.

28 LEGUINA-RUZZI A, PEREIRA J, PEREIRA-FLORES K, VALDERAS JP, MEZZANO D, VELARDE V, et al. Increased RhoA/Rho-Kinase Activity and Markers of Endothelial Dysfunction in Young Adult Subjects with Metabolic Syndrome. Metab Syndr Relat Disord. 2015; 13: 373-80. 10.1089/met.2015.0061.

29 ANDREASSEN AK, NORDOY I, SIMONSEN S, UELAND T, MULLER F, FROLAND SS, et al. Levels of circulating adhesion molecules in congestive heart failure and after heart transplantation. Am J Cardiol. 1998; 81: 604-8.

30 AUKRUST P, UELAND T, MULLER F, ANDREASSEN AK, NORDOY I, AAS H, et al. Elevated circulating levels of $\mathrm{C}-\mathrm{C}$ chemokines in patients with congestive heart failure. Circulation. 1998; 97: 1136-43.

31 SHANTSILA E, WRIGLEY BJ, BLANN AD, GILL PS, LIP GY. A contemporary view on endothelial function in heart failure. Eur J Heart Fail. 2012; 14: 873-81. 10.1093/eurjhf/ hfs066.

32 DJOHAN AH, SIA CH, LEE PS, POH KK. Endothelial Progenitor Cells in Heart Failure: an Authentic Expectation for Potential Future Use and a Lack of Universal Definition. J Cardiovasc Transl Res. 2018; 11: 393-402. 10.1007/s12265018-9810-4. 
33 ANDRE C, PIVER E, PERAULT R, BISSON A, PUCHEUX J, VERMES E, et al. Galectin-3 predicts response and outcomes after cardiac resynchronization therapy. J Transl Med. 2018; 16: 299. 10.1186/s12967-018-1675-4.

34 CHEN J, GARCIA EV, BAX JJ, ISKANDRIAN AE, BORGES-NETO S, SOMAN P. SPECT myocardial perfusion imaging for the assessment of left ventricular mechanical dyssynchrony. J Nucl Cardiol. 2011; 18: 685-94. 10.1007/ s12350-011-9392-x.

35 WARRINER DR, LAWFORD P, SHERIDAN PJ. Measures of endothelial dysfunction predict response to cardiac resynchronisation therapy. Open Heart. 2016; 3: e000391. 10.1136/openhrt-2015-000391.

36 MARTI CN, GHEORGHIADE M, KALOGEROPOULOS AP, GEORGIOPOULOU VV, QUYYUMI AA, BUTLER J. Endothelial dysfunction, arterial stiffness, and heart failure. J Am Coll Cardiol. 2012; 60: 1455-69. 10.1016/j. jacc.2011.11.082.

37 KUVIN JT, PATEL AR, SLINEY KA, PANDIAN NG, SHEFFY J, SCHNALL RP, et al. Assessment of peripheral vascular endothelial function with finger arterial pulse wave amplitude. Am Heart J. 2003; 146: 168-74. 10.1016/S00028703(03)00094-2.

38 MARTIN BJ, GURTU V, CHAN S, ANDERSON TJ. The relationship between peripheral arterial tonometry and classic measures of endothelial function. Vasc Med. 2013; 18: 13-8. $10.1177 / 1358863 X 12468194$.

39 OCARANZA MP, GABRIELLI L, MORA I, GARCIA L, MCNAB P, GODOY I, et al. Markedly increased Rho-kinase activity in circulating leukocytes in patients with chronic heart failure. Am Heart J. 2011; 161: 931-7. 10.1016/j. ahj.2011.01.024. 